\title{
Stili di vita nell'Italia della crisi: il cambiamento nelle abitudini insalubri secondo le condizioni lavorative
}

Simone Sarti ${ }^{1}$, Marco Terraneo ${ }^{2}$ e Mara Tognetti Bordogna ${ }^{2}$

Sessione 10 - Come promuovere salute nel lavoro? Strategie istituzionali, politiche organizzative e implicazioni per il sistema di welfare

Paper for the IX ESPAnet Italy Conference

"Welfare models and Varieties of Capitalism. The challenges to the socio-economic development in Italy and Europe"

Macerata, 22-24 September 2016 
Il paper costituisce una versione provvisoria e in corso di aggiornamenti. Si prega di non citare senza il consenso degli autori.

\title{
Stili di vita nell'Italia della crisi: il cambiamento nelle abitudini insalubri secondo le condizioni lavorative
}

\author{
Simone Sarti ${ }^{1}$, Marco Terraneo ${ }^{2}$ e Mara Tognetti Bordogna ${ }^{2}$ \\ ${ }^{1}$ Università degli Studi di Milano \\ ${ }^{2}$ Università degli Studi di Milano-Bicocca
}

\begin{abstract}
La salute è influenzata da una serie di fattori tra loro in interazione. Essi possono essere ricondotti a tre dimensioni: condizioni sociali, fattori biologici e caratteristiche contestuali o ecologiche. Un'ampia letteratura riconosce nelle condizioni strutturali un importante determinante del deterioramento delle condizioni di salute degli individui. Sono moltissimi gli studi che documentano un'influenza sulla salute delle tradizionali variabili di stratificazione sociale, come il titolo di studio e la condizione occupazionale. Tuttavia, sappiamo anche che il peggioramento delle condizioni socio-economiche si trasmette solo in parte in modo diretto. Sussistono infatti numerosi effetti trasversali e indipendenti dalla posizione socio-economica legati a stili di vita individuali e familiari. Descrivere le dinamiche di queste relazioni, anche alla luce dei cambiamenti a livello macro, come quelli imposti dalla crisi economica, appare un compito di centrale importanza. Alcuni autori, ad esempio, sostengono che fasi di recessione economica producano effetti ambivalenti sulle condizioni di salute (Karanikolos et al. 2013; Merino 2013; Suhrcke \& Stuckler 2012; Ruhm 2000). In questo senso si evidenzia che nei periodi di crisi alcuni comportamenti insalubri tendano a ridursi per una serie di ragioni contingenti, tra cui la minore disponibilità economica di cui gli individui beneficiano. Ad esempio, la crescita delle tasse sull'alcol e sul tabacco per necessità finanziarie avrebbero indotto una riduzione di questi comportamenti pericolosi per la salute. Ancora, si sarebbe osservata in molti paesi una diminuzione delle morti per incidenti stradali, poiché gli automobilisti si sarebbero indirizzati verso mezzi di trasporto meno costosi o avrebbero ridotto i loro viaggi.

D'altra parte, comprendere ed esplorare come le abitudini insalubri possano essersi modificate entro i diversi gruppi occupazionali con l'avvento della crisi può aiutare a mettere in atto pratiche di Work Health Promotion (WHP), ossia forme di promozione della salute attivate dai datori di lavoro, privati e pubblici, che consentono di attuare interventi di prevenzione mirati e di maggiore efficacia nei luoghi di lavoro.

Date queste premesse, in questo studio è nostro interesse mostrare i cambiamenti in alcuni fattori di rischio negli anni della crisi. Ciò sarà fatto impiegando i dati ISTAT Multiscopo Aspetti della vita quotidiana. Le analisi saranno condotte con tecniche di regressione multivariata gerarchica e tecniche di riduzione dei dati.

Tra $\mathrm{i}$ fattori di rischio epidemiologicamente validati l'attenzione è stata posta sui seguenti: 1) fumo (Gallus et al. 2015); 2) alcool (Ruhm 1995); 3) dieta (Merino 2013); 4) attività fisica (Karanikolos et al. 2013).

L'obiettivo più generale dello studio è dunque migliorare la comprensione dell'intricato sistema di relazioni tra variabili socio-economiche, stili di vita e salute, in modo da offrire conoscenze utili al decisore politico, al fine di predisporre interventi maggiormente informati di prevenzione primaria e secondaria sulla salute.
\end{abstract}


Inoltre, di una conoscenza più approfondita delle dinamiche in atto possono beneficiare i datori di lavoro, che in una prospettiva di WHP, saranno in grado di promuovere con più efficacia pratiche a sostegno di stili di vita sani.

Più in particolare lo scopo di questo lavoro è comprendere quali siano i percorsi attraverso cui gli svantaggi occupazionali possono tradursi in stili di vita insalubri e conseguentemente in peggiori condizioni di salute. Intervenire in modo perequativo, al fine di ridurre le disuguaglianze di salute, può significare sia agire sulla disuguaglianza sociale tout court, ma anche intervenire sugli snodi attraverso cui questa disuguaglianza si riverbera sulla salute.

\section{Keywords:}

stili di vita; disuguaglianze di salute; crisi economica; classe sociale; gruppi occupazionali 


\section{Introduzione}

Nello studio delle disuguaglianze di salute, la relazione tra posizione sociale, comportamenti insalubri e salute è ben documentata.

Come noto, tra i determinanti strutturali (WHO 2010) delle condizioni di salute, la classe sociale e la posizione occupazionale sono ritenuti fattori importanti per dar conto della posizione socioeconomica che gli individui occupano nella società. D'altra parte, sappiamo anche che un gradiente sociale per la salute esiste, ossia che la salute è progressivamente migliore più è alta la posizione socioeconomica delle persone (Marmot et al., 2012). Oltre ad un effetto diretto, i determinanti strutturali agiscono anche in modo indiretto sulla salute attraverso l'impatto che essi hanno su un insieme di determinanti intermediari della salute (WHO 2010). Infatti, è la posizione sociale che determina la differenza di esposizione e la vulnerabilità ai fattori intermediari che possono compromettere la salute. Tra i principali determinanti intermediari ci sono i fattori comportamentali, quali il fumo, la dieta, il consumo di alcol, l'esercizio fisico, fattori che agiscono sia proteggendo (come l'esercizio fisico) che danneggiando (come il fumo di sigaretta) la salute.

In questo lavoro ci domandiamo in che modo la crisi economica che ha colpito il nostro paese (e non solo) dal 2008 abbia avuto un impatto sulle condizioni di salute guardando alla relazione tra posizione occupazionale/classe sociale e comportamenti salubri o insalubri tenuti dagli individui. La letteratura scientifica che si è soffermata sulle conseguenze della crisi economica sulla salute ha mostrato posizioni spesso contrastanti per uno stesso outcome. Emerge, inoltre, come la crisi possa essere associata con risultati di salute negativi per alcuni, ma non per tutti, gli indicatori di salute (van Gool e Pearson, 2014). Da una parte, molti studiosi hanno sottolineato la forte e positiva associazione a livello individuale tra basso reddito, disoccupazione e cattiva salute come diretta conseguenza della crisi economica e finanziaria (Suhrcke e Stuckler, 2012; Sarti e Zella 2016). Sembra ben accertato, ad esempio, che durante i periodi di crisi il tasso di suicidi aumenta in un numero consistente di Paesi (Marmot e Bell, 2009; Falagas et al. 2009; Catalano et al. 2011; Economou et al. 2011). Al contrario, parecchi ricercatori hanno messo in luce che la recessione economica (per lo meno nel breve periodo) può migliorare lo stato di salute degli individui. In questo senso, si sostiene che nei periodi di crisi alcuni comportamenti insalubri tenderebbero a ridursi per una serie di ragioni contingenti, tra cui la minore disponibilità economica di cui gli individui beneficiano. Ad esempio, la crescita delle tasse sull'alcol e sul tabacco per recuperare risorse finanziarie da parte dello stato (Karanikolos et al. 2013) e, più in generale, un aumento dei prezzi di questi beni (Asgerisdottir et al. 2014), avrebbe indotto un calo dei comportamenti pericolosi per la salute. Inoltre, si sarebbe osservata in molti paesi una diminuzione delle morti per incidenti stradali (Suhrcke e Stuckler, 2012), poiché gli automobilisti si sarebbero indirizzati verso mezzi di trasporto più economici, o avrebbero ridotto i loro viaggi per risparmiare sui costi, o, ancora, per una parte di essi sarebbe svanita la necessità di utilizzare l'auto per lavoro visto che il lavoro l'hanno perso.

Il nostro interesse pertanto è quello di cercare di chiarire se e in che misura nel breve periodo la crisi economica abbia modificato in modo significativo gli stili di vita degli italiani, che sappiamo essere fortemente associati alle condizioni di salute degli individui. Nello specifico, tra $\mathrm{i}$ fattori di rischio epidemiologicamente validati l'attenzione è stata posta sui seguenti: a) fumo; b) alcool; c) dieta; d) attività fisica. 
Inoltre, proveremo a leggere gli eventuali cambiamenti negli stili di vita da una prospettiva che tenga in considerazione la posizione occupazionale/classe sociale che gli individui occupano nella gerarchia sociale. Infatti, non va dimenticato che le disuguaglianze di salute, per quanto riguarda natura, direzione, intensità e andamento temporale sono a svantaggio delle posizioni inferiori (Mackenbach et al., 2008; Costa, 2009) e, dunque, l'impatto della crisi potrebbe avere conseguenze differenti in virtù delle maggiori o minori risorse economiche, sociali e cognitive di cui gli individui dispongono.

\section{Letteratura empirica}

Come anticipato, in letteratura la relazione tra la crisi economica e gli stili di vita associati alla salute mostra risultati contrastanti. Inoltre, l'impatto del ciclo economico sui comportamenti sembra modificarsi in base alla posizione sociale che occupano gli individui.

Per molti autori i modelli di consumo relativi a fumo e alcol sono pro-ciclici, ossia si osserverebbe una diminuzione del consumo quando l'economia decresce e il tasso di disoccupazione aumenta (Henkel 2011; Asgerisdottir et al. 2014). Per quanto riguarda il consumo di alcol questi risultati sono confermati da Rhum e Black (2002), mentre Dee (2001), Davalos et al. (2012) e Xu e Kaestner (2010) trovano nei loro lavori che i problemi con l'alcol siano contro-ciclici, pertanto le persone aumenterebbero il consumo di alcol nei periodi di difficoltà economica. Ancora, Vilaplana et al. (2006) sostengono nel loro studio che il consumo di alcol non sia influenzato dal ciclo economico.

Se guardiamo al fumo, Ruhm (2005), Xu e Kaestner (2010), McClure et al. (2012) e Filippidis et al. (2014) suggeriscono che la prevalenza di fumatori diminuisce in associazione con la crisi economica, confermando l'andamento pro-ciclico di questo comportamento. D'altra parte, Charles e DeCicca (2008) mostrano che l'effetto del tasso di disoccupazione sul fumo (degli uomini) dipende dalla loro probabilità di essere disoccupati. Per i soggetti più vulnerabili alla disoccupazione, un più alto tasso di disoccupazione sarebbe associato a un maggior tasso di fumatori (pertanto si confermerebbe un andamento contro-ciclico), mentre per coloro che non si trovano in condizione di vulnerabilità (la maggioranza delle persone) il tasso di disoccupazione non sarebbe correlato con la quota di fumatori. In questa stessa direzione vanno i risultati di Gallus et al. (2015), secondo i quali la crisi economica del 2008 ha avuto un debole effetto sulla prevalenza di fumatori, sebbene questo esito sia il prodotto della relazione pro-ciclica osservata per gli occupati (minor numero di fumatori), compensata dalla relazione contro-ciclica trovata tra i disoccupati (maggior numero di fumatori).

Se spostiamo l'attenzione a quanto accade in l'Italia in relazione al consumo di alcol e fumo, come riportato da Costa et al. (2012), i dati PASSI mostrano nel periodo 200811 una riduzione della prevalenza di fumatori solo per le Asl del Centro d'Italia, mentre nel resto del paese l'andamento è compatibile con una sostanziale stabilità dell'abitudine negli anni della crisi. Inoltre, la stessa indagine segnala che i tentativi di smettere di fumare sarebbero in calo. Anche i dati DOXA (Costa et al. 2012) confermerebbero una diminuzione nella proporzione di fumatori tra il 2008 e il 2011. Sempre secondo i dati riportati da Costa et al. 2013, sia l'ISTAT che il sistema di sorveglianza PASSI, indicherebbero una diminuzione costante delle diverse forme di abuso di alcol, secondo un trend di lungo periodo, che si sarebbe però interrotto nei 
primi anni della crisi, tra il 2008 e il 2010, per poi riprendere dal 2011. D'altra parte, gli stessi autori sottolineano, come dato a cui prestare particolare attenzione, che con la crisi economica si sarebbe assistito a una tendenza all'aumento dei comportamenti a rischio legati al consumo di alcol tra i giovani (ad esempio una crescita del binge drinking).

Oltre al fumo e all'alcol, è stato spesso oggetto di indagine anche l'impatto della crisi sulla dieta seguita dagli individui. Come sappiamo, una cattiva alimentazione può avere significative conseguenze sulla salute. Sotto questo punto di vista, il lavoro di Ruhm (2000) mostra che il livello di disoccupazione non sembra modificare i comportamenti più salubri in termini di cibo consumato, quali il consumo di frutta e verdura. Dave e Kelly (2012), che distinguono tra cibi salubri e cibi insalubri, hanno analizzato se e in che misura il ciclo economico abbia avuto un effetto sul loro consumo. Per quanto riguarda i cibi insalubri (snack e cibo da fast-food) gli autori non osservano effetti statisticamente significativi, mentre notano un effetto pro-ciclico per i cibi salubri (frutta e verdura): un più alto rischio di disoccupazione sarebbe pertanto associato negativamente con questo ultimo tipo di consumo. Filippidis et. al. (2014) registrano che il consumo di almeno cinque porzioni di frutta e verdura al giorno (secondo le indicazioni della WHO) diminuisce significativamente durante la crisi tra i soggetti di più basso status socio-economico in Grecia. Ancora, sempre in tema di dieta la crisi sembra aver avuto un effetto negativo sull'adesione alla dieta mediterranea, che è noto essere storicamente associata con la buona salute (Willett et al. 1995; Sofi et al. 2008). Secondo Bonaccio et al. (2014), ad esempio, tra il 2007 e il 2010 si è assistito a un forte declino dell'adesione alla dieta mediterranea, una decrescita fortemente associata agli indicatori socio-economici (con i soggetti più istruiti e più ricchi più propensi a seguire questo tipo di dieta). D'altra parte, prima dell'inizio della crisi gli autori non avrebbero trovano nessuna associazione tra risorse disponibili e adesione alla dieta. Riscontri simili si trovano in una serie di studi condotti a Creta, che evidenziano la relazione tra crisi economica, posizione sociale e adesione alla dieta mediterranea (Karlen et al. 2008; Katsarou et al. 2010). Anche in questo caso, sarebbero gli individui che occupano le posizioni più svantaggiate ad aver abbandonato in misura più consistente l'adesione alla dieta mediterranea.

I dati dell'indagine multiscopo ISTAT riportati da Costa et al. (2012), evidenziano che, dopo un andamento altalenante, nel nostro Paese il consumo di frutta e verdura faccia registrare una contrazione consistente nel 2011 (ultimo dato disponibile), soprattutto nel Mezzogiorno.

Infine, un'ulteriore dimensione della relazione tra crisi economica e salute su cui intendiamo soffermare l'attenzione concerne l'attività fisica. Anche in questo caso, gli studi mostrano risultati contrastanti sull'effetto della crisi. Per alcuni, la crisi economica, con la conseguente crescita della disoccupazione o la riduzione dell'orario di lavoro, avrebbe aumentato il tempo libero da dedicare all'esercizio fisico. In questo senso Ruhm (2005) ha osservato che durante la temporanea crisi economica tra il 1987 e il 2000 l'attività fisica sarebbe cresciuta negli Stati Uniti. Questi risultati trovano parziale conferma in Colman e Dave (2013), che osservano l'impatto della recente recessione sui lavoratori e sui loro partner. Essi mostrano come la perdita di lavoro abbia aumentato l'esercizio fisico di tipo ricreativo degli individui studiati, confermando pertanto i dati di Ruhm (2005), ma che questo incremento non abbia compensato la decrescita di esercizio fisico svolta sul posto di lavoro, pertanto con una perdita netta in termini di attività fisica. Inoltre, gli autori osservano come la riduzione dell'attività fisica sia stata più consistente per le persone con status socioeconomico più basso, con le conseguenze di lungo termine che ciò comporta per la 
salute degli individui. Di segno opposto rispetto a Ruhm (2005) è invece la relazione osservata da Filippidis et al. (2014), secondi i quali in Grecia durante il periodo 200811, dunque nella prima fase della crisi economica, la prevalenza di alti livelli di attività fisica sarebbe cresciuta in tutti i gruppi socio-economici e demografici, con l'eccezione dei soggetti del gruppo socio-economico più alto.

Per quanto riguarda l'Italia, ci rifacciamo nuovamente ai dati riportati da Costa et al. (2012), secondo i quali i dati ISTAT mostrano che, con l'eccezione del Nord Italia, si assisterebbe a una flessione nella crescita della pratica sportiva continuativa, segnalando un rischio soprattutto per le aree più colpite dall'impoverimento.

\section{Ipotesi, dati e metodo}

Sulla base della precedente riflessione teorica siamo interessati in questo studio a descrivere l'andamento di alcuni fattori di rischio comportamentale negli anni della crisi tenendo in considerazione le condizioni socio-economiche degli italiani. Per soddisfare questo obiettivo sono stati impiegati i dati ISTAT Multiscopo Aspetti della Vita Quotidiana dal 2005 al 2013. La finestra temporale dovrebbe permettere di intercettare i possibili effetti della crisi economica iniziata nel 2008.

Innanzi tutto si è proceduto a selezionare i soggetti di età compresa tra i 30 e i 64 anni, al fine di considerare nelle analisi solo gli italiani che abbiano quasi certamente terminato gli studi e che non siano troppo anziani. Abbiamo optato per 64 anni come limite di età per evitare l'insorgenza di possibili problemi di selezione, in quanto, come noto, la mortalità prematura delle persone svantaggiate economicamente si fa più intensa per le fasce di popolazione anziana, per poi ricomporsi nell'anzianità avanzata (Willson et al. 2007).

All'interno dei questionari sono stati poi selezionati diversi indicatori considerati proxy affidabili dei comportamenti effettivamente tenuti dagli intervistati. Si è deciso di utilizzare i profili di rischio in forma dicotomica, ossia applicando delle soglie capaci di identificare soggetti certamente portatori di abitudini insalubri. Nello specifico, sono state riconosciute le seguenti condizioni come insalubri.

- Abitudine al fumo: l'intervistato dichiara di essere fumatore.

- Consumo intenso di alcool: l'intervistato dichiara di consumare almeno mezzo litro al giorno di una bevanda alcolica (birra, vino o altri alcolici).

- Condizione di obesità: l'intervistato ha un indice di massa corporea superiore a 30 .

- Dieta alimentare squilibrata: l'intervistato dichiara di consumare carne tutti i giorni, oppure di consumare meno di una volta a settimana frutta o verdura, oppure di usare oli non vegetali per cucinare.

- Nessuna attività fisica: l'intervistato dichiara di non esercitare nessuna attività fisica sul lavoro, in ambito domestico o volontaria.

Come si osserva dalla tabella 1 , che riporta la frequenza percentuale di individui che hanno tenuto comportamenti a rischio nell'arco temporale analizzato, con l'eccezione del consumo eccessivo di alcol che mostra un calo costante in tutto il periodo (dal 6,6\% nel 2005 al 3,5\% nel 2013), gli altri indicatori mostrano un andamento altalenante, con anni di diminuzione seguiti da anni in cui la quota è cresciuta e viceversa. In generale, sembra si possa affermare che rispetto al periodo pre-crisi la frequenza dei comportamenti insalubri sia aumentata negli anni inziali della crisi per poi ridursi nuovamente. 
Tabella 1. Frequenza percentuale degli indicatori di comportamenti a rischio per la salute.

\begin{tabular}{l|r|r|r|r|r|r|r|r|r|} 
& \multicolumn{1}{|c|}{2005} & \multicolumn{1}{|c|}{2006} & 2007 & $\mathbf{2 0 0 8}$ & 2009 & 2010 & 2011 & 2012 & 2013 \\
\hline Fumo & 25,9 & 27,2 & 26,6 & $\mathbf{2 6 , 7}$ & 27,5 & 27,2 & 26,6 & 26,2 & 25,2 \\
Alcool & 6,6 & 6,4 & 6,4 & $\mathbf{5 , 7}$ & 5,2 & 4,9 & 4,5 & 3,9 & 3,5 \\
Obesità (imc>30) & 10,4 & 10,7 & 10,7 & $\mathbf{1 0 , 2}$ & 10,5 & 10,3 & 10,3 & 10,4 & 10,3 \\
Dieta sbilanciata & 27,0 & 26,6 & 27,4 & $\mathbf{2 7 , 5}$ & 27,3 & 26,8 & 27,0 & 26,0 & 26,7 \\
Nessuna attività fisica & 13,3 & 14,2 & 13,7 & $\mathbf{1 4 , 0}$ & 14,1 & 15,8 & 14,5 & 14,7 & 14,5 \\
& & & & & & & & & \\
Totale* & 22873 & 22576 & 22090 & $\mathbf{2 2 5 2 6}$ & 21973 & 22580 & 22135 & 21628 & 9350
\end{tabular}

*È indicato il numero più basso di casi validi considerati tutti gli indicatori presi in esame.

Dal punto di vista delle variabili dipendenti, trattandosi il nostro di uno studio con finalità descrittive, si è deciso di realizzare una combinazione delle variabili strutturali al fine di identificare una serie di gruppi sociali definiti su base socio-economica.

Le variabili utilizzate nella costruzione di questi gruppi sono la condizione occupazionale, che distingue tra persone in cerca di lavoro (sia della prima occupazione che di una nuova occupazione), persone che svolgono attività non retribuite (per lo più casalinghe; ricordiamo che data la selezione dell'età effettuata in fase preliminare all'analisi gli studenti sono quasi del tutto assenti) e occupati; la posizione occupazionale, che distingue tra operai, piccola borghesia (che comprende lavoratori autonomi, commercianti, artigiani) classe media impiegatizia (che comprende impiegati, funzionari, quadri) e borghesia (che comprendere liberi professionisti, imprenditori, dirigenti) e il livello di istruzione, che distingue tra bassa istruzione, ossia coloro che possiedono un titolo di studio equivalente alla scuola dell'obbligo o meno, e alta istruzione, equivalente a un titolo di studio pari al diploma di scuola secondaria o superiore). Nella Tabella 2 l'andamento della percentuale dei soggetti in cerca di lavoro mostra con buona evidenza l'effetto strutturale della crisi.

Tabella 2. Percentuali secondo la condizione occupazionale tra il 2005 e il 2013 ( $L=$ bassa istruzione, $\mathrm{H}=$ alta istruzione; $\mathrm{OPE}=$ Operai, $\mathrm{PB}=$ Piccola borghesia, $\mathrm{CMI}=\mathrm{Classe}$ media impiegatizia, $\mathrm{BOR}=$ Borghesia).

\begin{tabular}{l|r|r|r|r|r|r|r|r|r|} 
& \multicolumn{1}{|c|}{2005} & \multicolumn{1}{c|}{2006} & \multicolumn{1}{c|}{2007} & $\mathbf{2 0 0 8}$ & 2009 & 2010 & 2011 & \multicolumn{1}{c}{2012} & 2013 \\
\hline In cerca di lavoro & 4,8 & 5,7 & 5,5 & $\mathbf{7 , 2}$ & 8,9 & 9,9 & 10,2 & 11,2 & 13,2 \\
Non lavoro retribuito & 19,8 & 19,3 & 18,3 & $\mathbf{1 7 , 9}$ & 19,0 & 18,0 & 17,3 & 15,7 & 15,9 \\
Ritirato dal lavoro & 10,7 & 10,5 & 10,5 & $\mathbf{1 0 , 9}$ & 9,4 & 9,6 & 9,2 & 8,5 & 7,2 \\
Occupati OPE-L & 16,8 & 16,6 & 17,1 & $\mathbf{1 5 , 8}$ & 15,3 & 14,2 & 15,2 & 15,3 & 12,0 \\
Occupati OPE-H & 3,5 & 3,8 & 3,8 & $\mathbf{4 , 3}$ & 4,2 & 4,4 & 4,7 & 5,5 & 8,1 \\
Occupati PB -L & 6,1 & 6,3 & 6,2 & $\mathbf{6 , 2}$ & 5,0 & 5,4 & 5,0 & 4,8 & 2,9 \\
Occupati PB-H & 19,4 & 19,1 & 19,8 & $\mathbf{1 9 , 6}$ & 18,8 & 19,9 & 19,5 & 20,0 & 22,2 \\
Occupati CMI-L & 7,5 & 6,9 & 6,8 & $\mathbf{7 , 0}$ & 7,0 & 6,5 & 6,3 & 6,4 & 5,1 \\
Occupati CMI-H & 2,9 & 3,1 & 3,2 & $\mathbf{3 , 2}$ & 3,2 & 3,6 & 3,8 & 3,7 & 5,7 \\
Occupati BOR-L & 2,3 & 2,6 & 2,4 & $\mathbf{1 , 7}$ & 2,2 & 1,5 & 1,8 & 1,6 &, 9 \\
Occupati BOR-H & 6,1 & 6,0 & 6,5 & $\mathbf{6 , 1}$ & 7,0 & 7,0 & 7,1 & 7,1 & 6,8 \\
Totale & 24271 & 24029 & 23741 & $\mathbf{2 4 0 8 5}$ & 23671 & 24045 & 23463 & 22763 & 9822
\end{tabular}

Per indagare in modo rigoroso le variazioni temporali si è deciso di applicare dei modelli di regressione binomiale multilivello (Snijders e Bosker 2011). I modelli sono realizzati su tre livelli $(\mathrm{i}, \mathrm{j}, \mathrm{k})$. Al primo livello si trovano gli individui intervistati 
( $\mathrm{I}=201233$ unità), al secondo livello le famiglie ( $\mathrm{J}=19720$ unità), al terzo livello le unità spazio-temporali, costituite dalla combinazione dell'area geografica (nord, centro e sud e gli anni, per un totale di 27 contesti). La combinazione tra unità geografiche e temporali (gli anni) si è resa necessaria al fine di ottenere un numero di unità di terzo livello adeguato. ${ }^{1}$

La distinzione di tre livelli permette di controllare l'endogeneità familiare (l'autocorrelazione presente negli individui appartenenti alla stessa famiglia). Inoltre, realizzando dei modelli random slope su alcuni dei regressori è stato possibile distinguere la variabilità temporale complessiva (ossia l'andamento generale del trend, tenuto conto delle variabili impiegate nel modello) dall'andamento del comportamento di rischio su specifiche categorie sociali.

Le variabili di controllo impiegate a livello individuale sono l'età, espressa in sette classi ${ }^{2}$, e il genere.

\section{Risultati}

I risultati generali delle analisi sono mostrati nella Tabella 3.

Essi mostrano il noto gradiente tante volte individuato negli studi sulle disuguaglianze sociali nella salute. La propensione al rischio è tendenzialmente più marcata per le categorie sociali socio-economicamente più svantaggiate sul mercato del lavoro in termini di capitale culturale, economico e sociale (Della Bella et al. 2011; Mackenbach et al 2008; Sarti 2006). In particolare i soggetti in cerca di lavoro, sono tra coloro maggiormente esposti al consumo di tabacco, all'alcol e all'obesità. Gli operai con bassi livelli di istruzione, e verosimilmente, con mansioni lavorative più esecutive, sono più esposti al fumo, all'alcol e ad un'alimentazione squilibrata. In condizioni svantaggio simile vi sono gli impiegati a bassa istruzione. Al contrario, in mostrano propensioni vantaggiose, con l'esclusione dell'attività fisica, la piccola borghesia e la borghesia con alti livelli di istruzione.

Come anticipato ci concentreremo sulla categoria dei soggetti in cerca di lavoro che è quella che maggiormente, anche se non esclusivamente, potrebbe aver risentito degli effetti della crisi e che è andando ingrossandosi sensibilmente nel periodo considerato. Le analisi condotte hanno infatti mostrato che per le categorie di occupati non vi sono state variazioni né statisticamente significative, né di qualche interesse dal punto di vista dell'andamento dei trend. Viceversa solo la categoria dei disoccupati ha mostrato indicazioni di cambiamenti negli anni della crisi degni di interesse. Per questa ragione nella Tabella 3 è riportata la covarianza tra regressore e variazione spazio-temporale solo quando si è rivelata di un qualche interesse (coefficienti random slope).

\footnotetext{
${ }^{1}$ Come noto il numero dovrebbe essere almeno superiore a 20 per soddisfare la normalità nella distribuzione dei residui (Bryan e Jenkins, 2013).

${ }^{2}$ Le classi di età impiegate sono le seguenti: $30-34,35-39,40-44,45-49,50-54,55-59$ e $60-64$. Si è preferito impiegare l'età come variabile categoriale perché alcuni comportamenti hanno una relazione non lineare con l'età.
} 
Tabella 3. Propensioni ai diversi comportamenti insalubri secondo la condizione occupazionale degli intervistati. Risultati del modello di regressione binomiale multilivello espressi in logit. Tra parentesi gli errori standard.

\begin{tabular}{|c|c|c|c|c|c|}
\hline & FUMO & ALCOL & OBESITÀ & DIETA & ATT.FISICA \\
\hline Costante (intercetta) & $-1.394(0.027)$ & $-4.892(0.074)$ & $-2.810(0.039)$ & $-0.918(0.029)$ & $-1.922(0.066)$ \\
\hline \multicolumn{6}{|l|}{$\begin{array}{l}\text { Cond.occupazionale } \\
\text { (rif. "non lavoro") }\end{array}$} \\
\hline In cerca di lavoro & $0.563(0.031)^{+}$ & $0.305(0.064)$ & $-0.099(0.040)^{+}$ & $0.066(0.025)$ & $0.249(0.031)^{+}$ \\
\hline Ritirato dal lavoro & $0.129(0.027)$ & $0.217(0.062)$ & $-0.163(0.031)$ & $-0.666(0.028)$ & $0.656(0.035)$ \\
\hline Operai-low & $0.584(0.021)$ & $0.458(0.058)$ & $-0.165(0.036)^{+}$ & $0.189(0.021)$ & $-0.458(0.031)$ \\
\hline Operai-high & $0.307(0.030)$ & $0.093(0.075)$ & $-0.377(0.046)$ & $-0.018(0.031)$ & $0.084(0.039)$ \\
\hline Pb-low & $0.391(0.028)$ & $0.013(0.071)$ & $-0.296(0.038)$ & $0.007(0.029)$ & $0.514(0.034)$ \\
\hline Pb-high & $0.013(0.021)$ & $-0.406(0.061)$ & $-0.726(0.029)$ & $-0.286(0.021)$ & $0.875(0.025)$ \\
\hline Cmi-low & $0.482(0.026)$ & $0.516(0.062)$ & $-0.186(0.035)$ & $0.148(0.027)$ & $-0.408(0.040)$ \\
\hline Cmi-high & $0.299(0.033)$ & $0.198(0.076)$ & $-0.480(0.050)$ & $-0.101(0.034)$ & $0.446(0.040)$ \\
\hline Bor-low & $0.378(0.041)$ & $0.365(0.081)$ & $-0.105(0.054)$ & $0.206(0.042)$ & $0.100(0.055)$ \\
\hline Bor-high & $0.128(0.027)$ & $-0.245(0.069)$ & $-0.662(0.040)$ & $-0.284(0.028)$ & $0.917(0.031)$ \\
\hline \multicolumn{6}{|l|}{ Variabili di controllo } \\
\hline \multicolumn{6}{|l|}{ Genere (rif. Femmine) } \\
\hline Maschi & $0.507(0.012)$ & $2.002(0.036)$ & $0.398(0.018)$ & $0.476(0.012)$ & $0.466(0.015)$ \\
\hline \multicolumn{6}{|l|}{ Età (rif. 30-34) } \\
\hline $35-39$ & $-0.097(0.020)$ & $0.095(0.048)$ & $0.295(0.036)$ & $-0.119(0.020)$ & $-0.253(0.023)$ \\
\hline $40-44$ & $-0.106(0.020)$ & $0.249(0.046)$ & $0.492(0.035)$ & $-0.240(0.020)$ & $-0.342(0.023)$ \\
\hline $45-49$ & $-0.095(0.020)$ & $0.477(0.046)$ & $0.796(0.034)$ & $-0.322(0.021)$ & $-0.481(0.024)$ \\
\hline $50-54$ & $-0.061(0.021)$ & $0.661(0.045)$ & $0.969(0.034)$ & $-0.415(0.022)$ & $-0.638(0.026)$ \\
\hline $55-59$ & $-0.252(0.022)$ & $0.736(0.046)$ & $1.058(0.034)$ & $-0.501(0.023)$ & $-0.781(0.027)$ \\
\hline $60-64$ & $-0.421(0.026)$ & $0.855(0.051)$ & $1.119(0.037)$ & $-0.582(0.027)$ & $-0.971(0.034)$ \\
\hline Cov (in cerca di lavoro) & $-0.009(0.003)$ & - & $-0.012(0.005)$ & - & $-0.022(0.009)$ \\
\hline Cov (operai low) & - & - & $-0.007(0.004)$ & - & - \\
\hline Var 3 & $0.008(0.003)$ & $0.054(0.016)$ & $0.012(0.004)$ & $0.012(0.003)$ & $0.101(0.028)$ \\
\hline Var 2 & $0.510(0.015)$ & $0.970(0.050)$ & $0.477(0.027)$ & $0.782(0.016)$ & $0.548(0.021)$ \\
\hline $\operatorname{Var} 1^{3}$ & 3.29 & 3.29 & 3.29 & 3.29 & 3.29 \\
\hline
\end{tabular}

Procediamo entrando nel dettaglio dell'analisi dei residui su base geografica e temporale al fine di evidenziare i cambiamenti intercorsi dal 2005 al 2013 nelle abitudini insalubri. Le analisi sono distinte per i cinque indicatori adottati. Gli intervalli di confidenza sono stati inseriti solo laddove sono stati ritenuti utili nella descrizione del trend.

\section{Abitudine al fumo}

La prima analisi effettuata riguarda l'abitudine al fumo.

Grafici 1A. Andamento temporale nella propensione ad essere fumatori: residui per area geografica (espressi come logit). 1B. Andamento temporale per i soggetti in cerca di lavoro.

\footnotetext{
${ }^{3}$ Le variabili dicotomiche dipendenti possono essere considerate come variabili continue ricodificate sulla base di una soglia definita arbitrariamente. In tal caso il valore della varianza al primo livello viene fissato a 3.29. Per approfondimenti si veda: Snijders and Bosker (2011).
} 

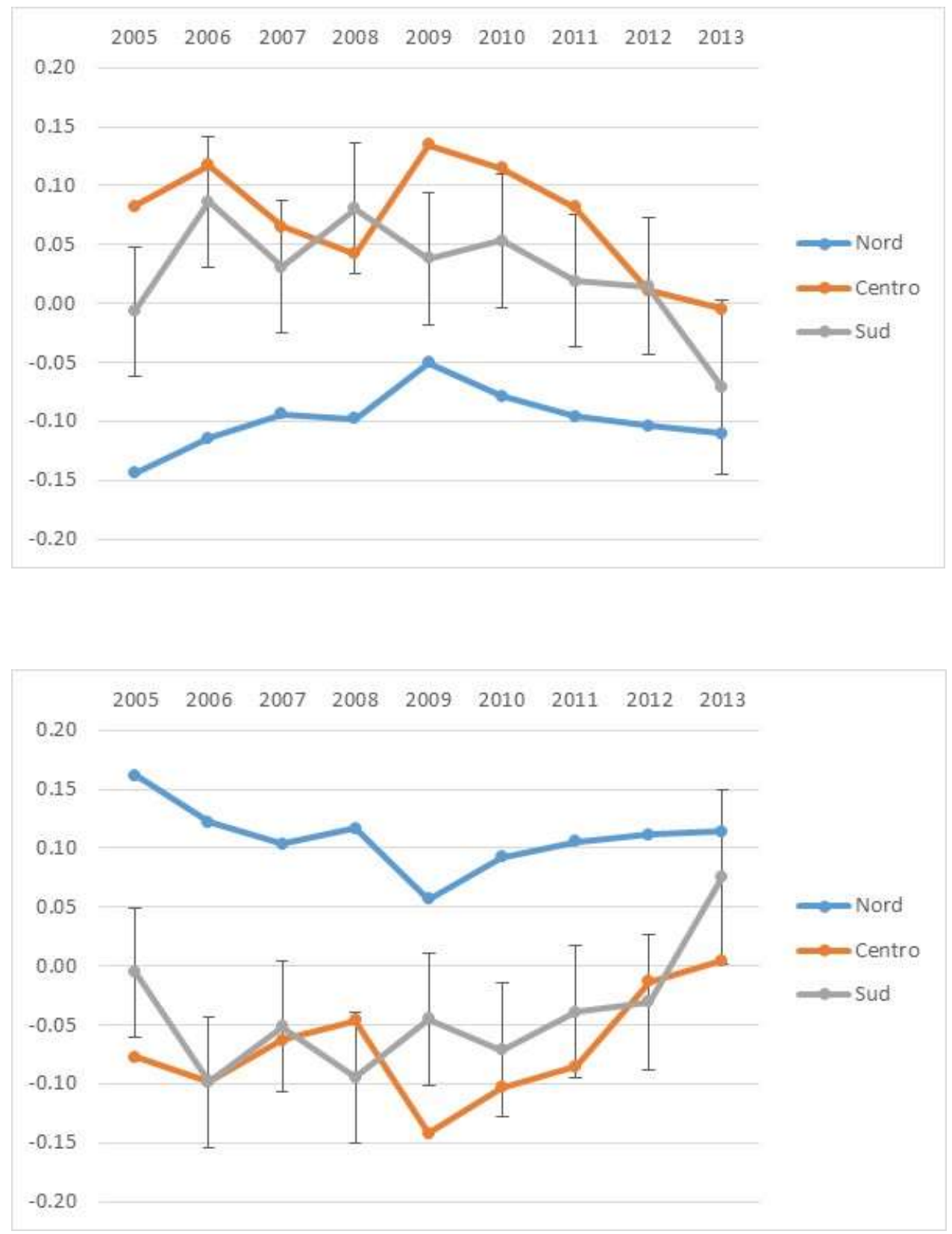

Le analisi non mostrano variazioni significative, anche se il 2013 suggerisce un decremento rilevante nella propensione generale al fumo al sud, e contemporaneamente un incremento degno di nota, ancora al sud, da parte dei soggetti in cerca di lavoro.

\section{Obesità}

Grafici 2A. Andamento temporale nella propensione ad essere obesi: residui per area geografica (espressi come logit). 2B. Andamento temporale per i soggetti in cerca di lavoro. 2C. Andamento temporale per gli operai con basso livello di istruzione. 


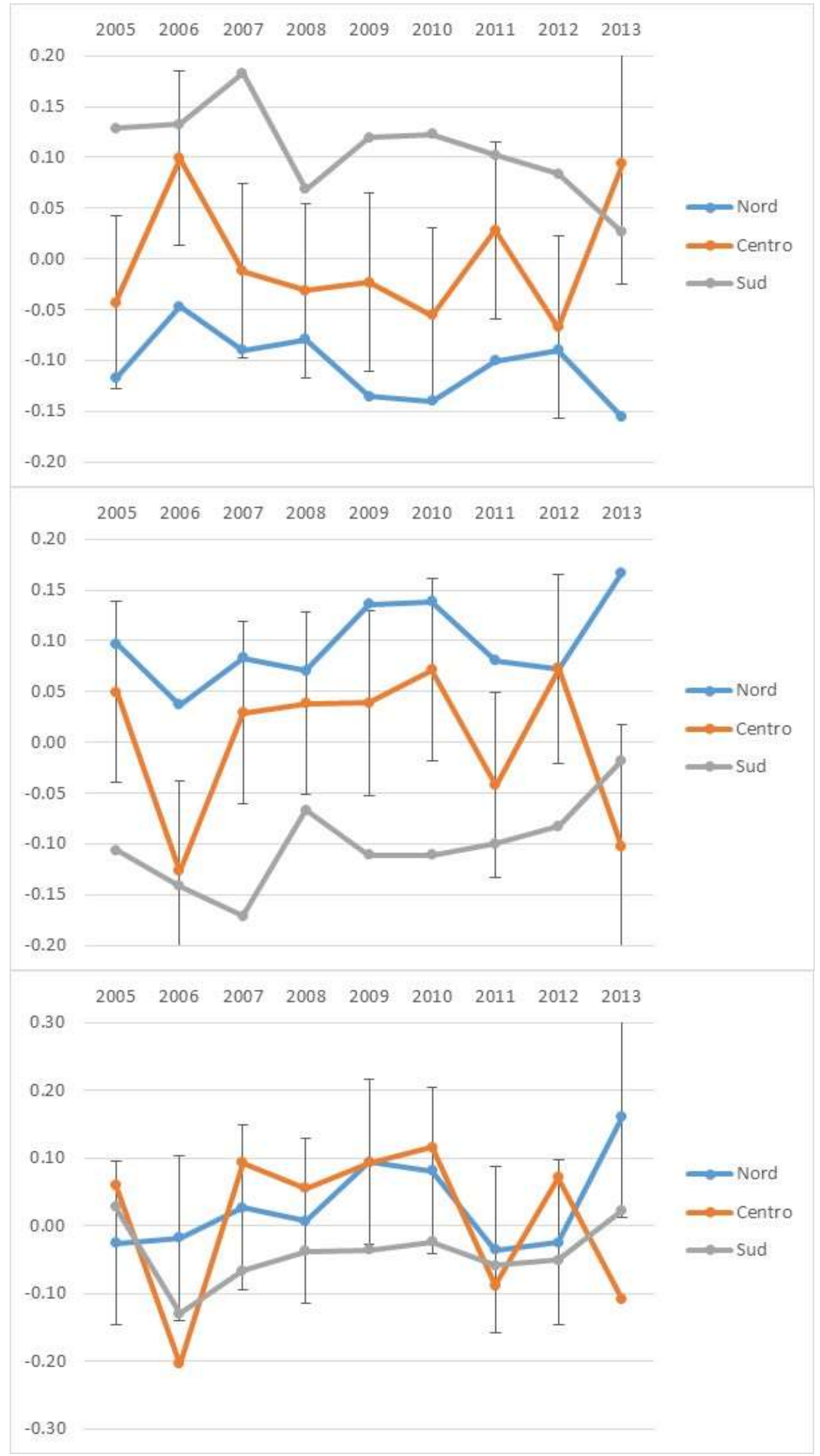

Le analisi dei residui rispetto all'obesità, ossia rispetto ai soggetti con indice di massa corporea superiore a 30 (secondo la definizione convenzionale), mostrano trend 
complessivamente non significativi. Tuttavia, come per il fumo, il 2013 suggerisce alcune variazioni degne di nota. Mentre complessivamente la propensione all'obesità sembra aumentare nelle regioni centrali d'Italia, essa tuttavia parrebbe diminuire leggermente per i soggetti in cerca di lavoro e per gli operai a bassa istruzione. Allo stesso tempo in queste categorie sociali sembrerebbe avviarsi una tendenza opposta al nord, ossia di un leggero aumento.

\section{Attività fisica}

Per ciò che concerne il rischio legato all'assenza di attività física, l'analisi temporale mostra una sostanziale stabilità complessiva, mentre un incremento sostanzialmente importante per i soggetti in cerca di lavoro al sud.

Purtroppo la stima degli intervalli di confidenza risulta problematica quindi non è possibile rappresentare graficamente la significatività dei coefficienti.

Grafici 3A. Andamento temporale nella propensione a non tenere nessuna attività fisica: residui per area geografica (espressi come logit). 3B. Andamento temporale per i soggetti in cerca di lavoro.

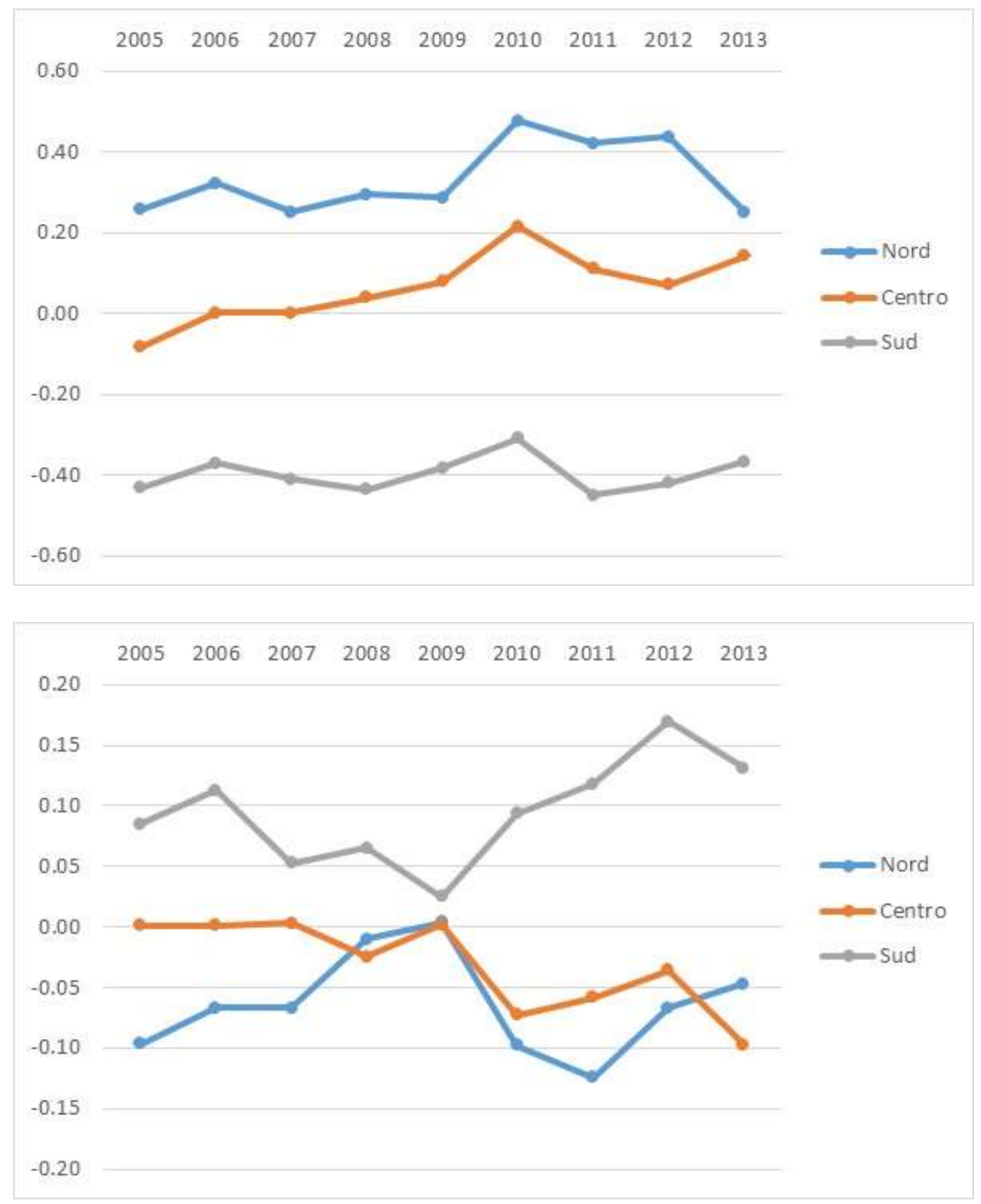




\section{Consumo di alcol e dieta alimentare.}

Per quanto riguarda il consumo di alcol e la dieta alimentare non emergono trend significativi rispetto a nessuna delle categorie utilizzate. Riportiamo solo i trend dei residui dell'intercetta.

Grafico 4. Andamento temporale nella propensione al consumo elevato di alcol: residui per area geografica (espressi come logit).

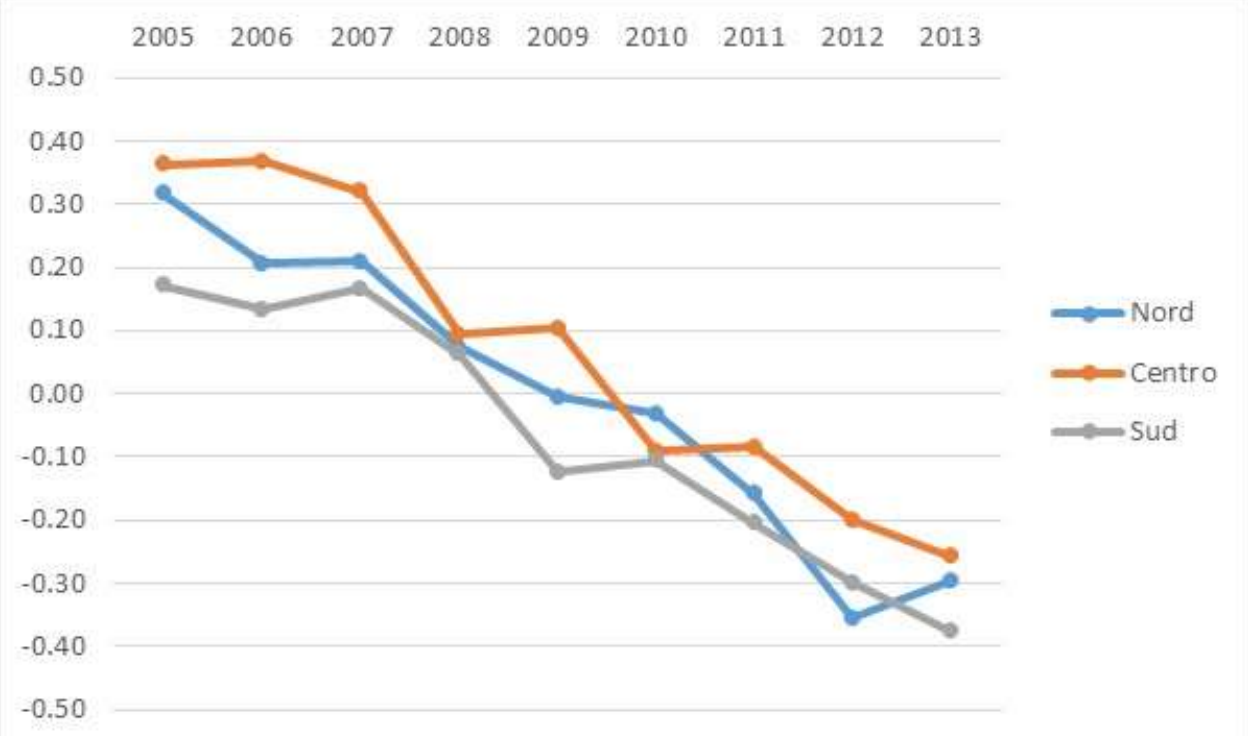

Grafico 5. Andamento temporale nella propensione a tenere una dieta alimentare sbilanciata: residui per area geografica (espressi come logit).

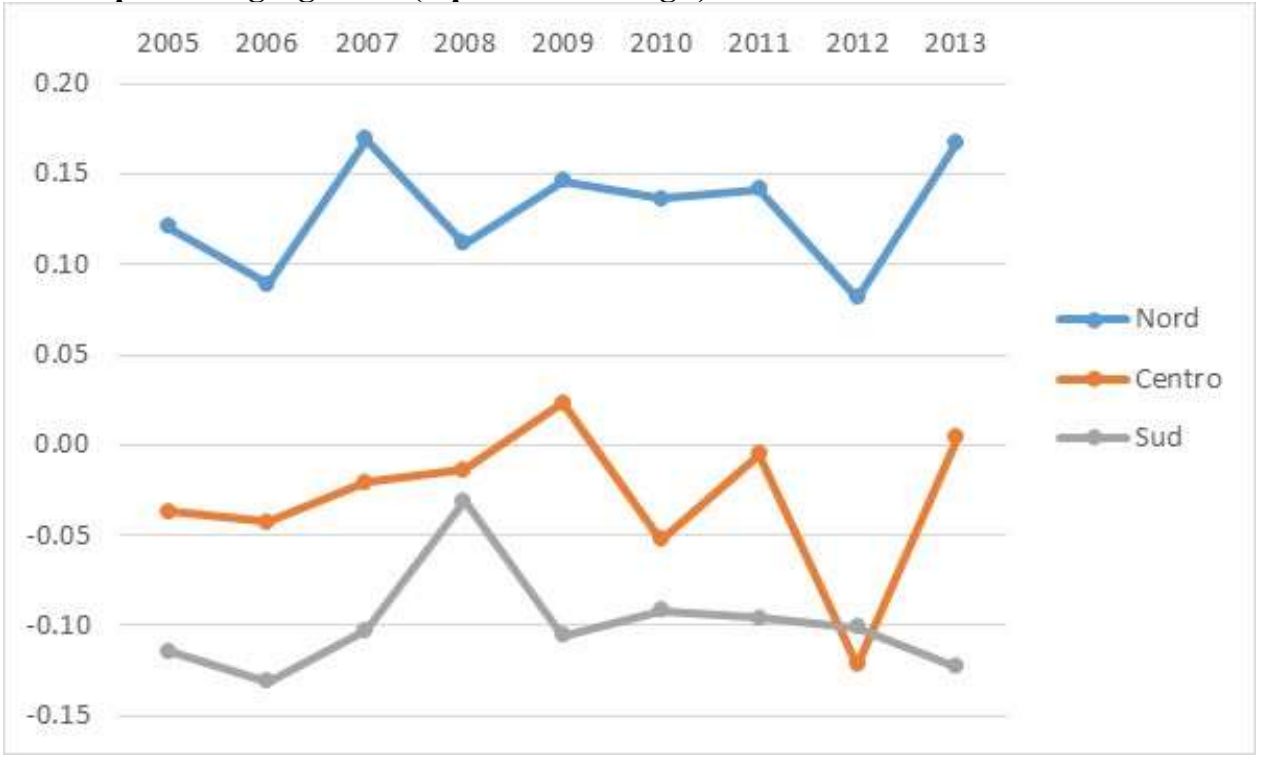

\section{Discussione e conclusioni}

In questo lavoro ci siamo proposti di descrivere l'andamento di alcuni fattori di rischio comportamentali legati alla salute.

Esiste infatti un dibattito tra gli studiosi della salute sugli effetti della crisi su questi comportamenti. Da un lato si afferma l'effetto paradosso della crisi che ridurrebbe alcune abitudini rischiose per diverse ragioni (calo dei consumi, minori spostamenti, 
eccetera), dall'altro si evidenzia come invece queste tendono ad aumentare (aumento stress e comportamenti autolesivi, De Vogli et al. 2013).

Per quanto riguarda il caso italiano le nostre analisi si sono concentrate sulla fascia lavorativa da 30 a 64 anni, particolarmente colpita dalla crisi socio-economica (cfr. Sarti e Vitalini 2016) relativamente a cinque fattori di rischio ampiamente trattati in letteratura: abitudine al fumo, consumo elevato di alcol, obesità, dieta sbilanciata $\mathrm{e}$ attività fisica.

Per quanto riguarda alcol e dieta alimentare non sono emerse variazioni degne di nota. Per l'alcol si registra una tendenziale diminuzione nella propensione a consumare eccessivamente quantità di alcol (almeno mezzo litro al giorno di una bevanda a base alcolica), come del resto già evidenziato da Costa e colleghi (2012), tendenza ben visibile dai trend descrittivi in Tabella 1.

Per la dieta alimentare non si registrano cambiamenti di alcun genere. Alcune categorie occupazionali sono tendenzialmente più esposte al rischio di diete sbilanciate, ma questa relazione non è cambiata dal 2005 al 2013. In particolare tutti gli occupati con basso livello di istruzione risultano più esposti (operai, impiegati e imprenditori).

Per il consumo di tabacco il trend generale mostra una lieve diminuzione e contemporaneamente non si evidenziano cambiamenti importanti nelle categorie occupazionali. L'unica categoria che mostra un trend significativo è quella dei soggetti in cerca di lavoro. In particolare pare sussistere, anche se non statisticamente significativo, un aumento della propensione al fumo tra i disoccupati del sud. Ricordiamo che la crisi socio-economica ha colpito in modo particolarmente duro le regioni del Mezzogiorno.

Tra i diversi indicatori l'obesità è quella che presenta il trend più intricato da leggere. I modelli presentano un effetto significativo (vedi covarianze nella Tabella 3) tuttavia non emergono direzioni sostanzialmente chiare nei trend.

Riguardo all'attività fisica si evidenzia, successivamente al 2008, 1'aumento della propensione al rischio di non svolgere alcuna attività fisica. Possiamo ipotizzare in tal senso una relazione con l'aumento del numero di disoccupati. In effetti, anche se $\mathrm{i}$ dati utilizzati, di natura cross-sectional non permettono di provarlo, l'aumento dell'inattività fisica è imputabile in buona misura alle regioni meridionali (si veda il Grafico 3B).

D'altra parte va ricordato che la propensione ai comportamenti a rischio è più alta per le categorie più vulnerabili, come $\mathrm{i}$ disoccupati, rispetto a quelle che occupano le posizioni più elevate nella gerarchia sociale. Questo dato non sembra essersi modificato con l'arrivo della crisi. Ciò significa che sebbene dalle nostre analisi non emerga sempre in modo evidente un cambiamento di comportamento all'interno delle diverse categorie nel corso del tempo, il fatto che la crisi abbia prodotto uno spostamento di una quota significa di soggetti tra le categorie, nello specifico dagli occupati ai disoccupati, potrebbe comportare che in termini assoluti il numero di individui con abitudini insalubri sia cresciuto negli ultimi anni. Se questo fosse vero, le conseguenze per la salute degli italiani sarebbero da non sottovalutare.

Concludendo, il tema degli effetti della crisi sui comportamenti insalubri si mostra decisamente articolato. Complessivamente gli effetti in Italia appaiono piuttosto modesti. Laddove alcuni segnali si presentano sostanzialmente degni di nota (anche se non statisticamente significativi), le indicazioni corroborano in modo più sostenuto l'idea di un peggioramento dell'esposizione al rischio da parte dei soggetti in cerca di lavoro (soprattutto rispetto a fumo e attività fisica). Mentre per gli occupati permangono le condizioni di svantaggio strutturale già presenti prima della crisi. 
Approfondimenti e conferme di questi risultati possono arrivare dall'analisi di banche dati sui consumi (Indagine sui consumi delle famiglie italiane) oppure dall'utilizzo di dati panel (ITA-SILC).

L'utilizzo di questi database costituisce il prossimo passo di questo studio.

Un ulteriore idea è di mettere in relazione la propensione a questi fattori di rischio in un modello di equazioni strutturali dove testare i nessi tra soddisfazione di alcuni aspetti della vita. Ipotizzando che il peggioramento delle condizioni socioeconomiche abbia un effetto diretto sulla soddisfazione e indiretto sui comportamenti. 


\section{Bibliografia}

Asgeirdottir, T.L., Corman, H., Noonan, K., Olafsdottir, P. e Reichman, N.E. (2014). Was the economic crisis of 2008 good for Icelanders? Impact on health behaviors. Economics and Human Biology, 13: 1-19.

Bonaccio et al. (2014). Decline of the Mediterranean diet at a time of economic crisis. Results from the Moli-sani study. Nutrition, Metabolism and Cardiovascular Disease, 24: 853-860

Bryan, M.L. e Jenkins, S.P. (2013). Regression analysis of country effects using multilevel data: a cautionary tale. ISER 2013e2014 (working paper series).

Catalano, R.R., Goldman-Mellor, S., Saxton, K., et al. (2011). The health effects of economic decline. Ann Rev Public Health 2011, 32:431-50.

Charles, K.K., DeCicca, P. (2008). Local labor market fluctuations and health: is there a connection and for whom? Journal of Health Economics, 27(6): 1532-1550.

Colman, G. e Dave, D. (2013). Exercise, physical activity, and exertion over the business cycle. Social Science \& Medicine, 93: 11-20. doi: http://dx.doi.org/10.1016/j.socscimed.2013.05.032

Costa, G. (2009). Le disuguaglianze di salute: una sfida per le discipline che si occupano di valutazione delle politiche, in Brandolini A., Schizzerotto A. e Saraceno C. (a cura di ). Dimensioni della disuguaglianza in Italia: povertà, salute, abitazione, Bologna, il Mulino.

Costa, G. et al. (2012). Gli indicatori di salute ai tempi della crisi in Italia, Epidemiologia \& Prevenzione, 36 (3), 337-366.

Costa, G., Marra M. e Salmaso S. (2013). La salute ai tempi della crisi, in Giansini, G. F., Nicelli A. L., Trabucchi, M. e Vanara F. (2013). Rapporto Sanità 2013. Sistema sanitario e sviluppo del Paese: alcune specificità in tempo di crisi, Fondazione Smith Kline, Bologna, il Mulino.

Davalos, M., Fang, H., French, M.T. (2012). Easing the pain of an economic downturn: macroeconomic conditions and excessive alcohol consumption. Health Economics, 21 (11): 1318-1335.

Dave, D.M., Kelly, I.R. (2012). How does the business cycle affect eating habits? Social Science \& Medicine, 74 (2): 254-262.

De Vogli R., Marmot M. e Stuckler D. (2013), Excess suicides and attempted suicides in Italy attributable to the great recession, Journal of epidemiology and community health, 67(4): 378-379.

Dee,T.S. (2001). Alcohol abuse and economic conditions: evidence from repeated cross-sections of individual-level data. Health Economics 10 (3): 257-270.

Della Bella S., Sarti S., Lucchini M. e Bordogna M.T. (2011), A comparative analysis of inequality in health across Europe, Sociological Research Online, 16(4), 7.

Economou, M., Madianos, M., Theleritis, C., et al. (2011). Increased suicidality amid economic crisis in Greece. Lancet, 378:1459.

Falagas, M.E., Vouloumanou, E.K., Mavros, M.N., et al. (2009). Economic crises and mortality: a review of the literature. Intl J Clin Pract, 63:1128-35.

Filippidis F.T., Schoretsaniti S., Dimitrakaki C., Vardavas C.I., Behrakis P., Connolly G.N. e Tountas Y. (2014). Trends in cardiovascular risk factors in Greece before and during the financial crisis: the impact of social disparities. European Journal of Public Health, 24: 974-979

Gallus, S., Ghislandi, S., \& Muttarak, R. (2015). Effects of the economic crisis on 
smoking prevalence and number of smokers in the USA. Tobacco control, 24(1): 8288 .

van Gool, K. E Pearson, M. (2014). Health, Austerity and Econiomic Crisis. Assessing the short-term impact in OECD countries. OECD Health Working Paper n.76, OECD Publishing.

Henkel, D. (2011). Unemployment and substance use: a review of the literature (1990-2010). Current drug abuse reviews, 4(1), 4.

Karanikolos, M., Mladovsky, P., Cylus, J., Thomson, S., Basu, S., Stuckler, D. e McKee, M. (2013). Financial crisis, austerity, and health in Europe. The Lancet, 381(9874), 1323-1331.

Karlen, J., Lowert, Y., Chatziarsenis,M., Faith-Magnusson, K., Faresjo, T. (2008).

Are children from Crete abandoning a Mediterranean diet?. Rural and Remote Health, 8:1034.

Katsarou, A, Tyrovolas, S., Psaltopoulou, T., Zeimbekis, A., Tsakountakis, N., Bountziouka, V., Gotsis, E., Metallinos, G., Polychronopoulos, E., Lionis, C. (2010). Socio-economic status, place of residence and dietary habits among the elderly: the Mediterranean islands study. Public Health Nutrition,13:1614-1621.

Mackenbach, J.P., Stirbu, I., Roskam, A.J., Schaap, M.M., Menvielle, G., Leinsalu, M., Kunst, A.E., European Union Working Group on Socioeconomic Inequalities in Health. 2008 Socioeconomic inequalities in health in 22 European countries. N Engl J Med, 358: 2468-2481

Marmot, M., Allen, J., Bell, R., Bloomer, E., \& Goldblatt, P. (2016). WHO European review of social determinants of health and the health divide. The Lancet, 380(9846), pp. 1011-1029. doi:10.1016/S0140-6736(12)61228-8

Marmot, M. G., e Bell, R. (2009). How will the financial crisis affect health? British Medical Journal, 338, b1314.

McClure, C.B., Valdimarsdóttir, U.A., Hauksdóttir, A., e Kawachi, I. (2012). Economic crisis and smoking behaviour: prospective cohort study in Iceland. BMJ open, 2(5).

Ruhm, C.J., (2000). Are recessions good for your health? The Quarterly Journal of Economics 115 (2) 617-650.

Ruhm, C.J. (2005). Healthy living in hard times. Journal of Health Economics, 24 (2): 341-363.

Ruhm C, Black W. (2002). Does drinking really decreases in bad times? Journal of Health Economics, 21: 659-78.

Sarti S. (2006). La classe sociale, in buona salute. Polis, XX, 3: 317-346.

Sarti, S., e Zella, S. (2016). Changes in the labour market and health inequalities during the years of the recent economic downturn in Italy. Social Science Research, 57, 116-132.

Sarti, S. e Vitalini, A. (2016). La salute degli italiani prima e dopo la crisi economica (2005-2013): alcune evidenze empiriche sulle categorie sociali a maggior rischio di impatto, Quaderni di Sociologia (Forthcoming).

Snijders, T. A., \& Bosker, R. J. (2011). Multilevel Analysis: An Introduction to Basic and Advanced Multilevel Modeling. SAGE.

Sofi, F., Cesari, F., Abbate, R., Gensini, G.F. e Casini A. (2008). Adherence to Mediterranean diet and health status: meta-analysis. BMJ 2008;337:a1344 doi: http://dx.doi.org/10.1136/bmj.a1344.

Suhrcke, M. e Stuckler, D. (2012). Will the recession be bad for our health? It 
depends. Social Science \& Medicine, 74: 647-653.

Vilaplana, C., Labeaga, J.M. e Jime'nez-Martı'n, S. (2006). Further evidence about alcohol consumption and the business cycle. Working Paper. 2006-06, FEDEA.

WHO. 2010. A conceptual framework for action on the social determinants of health. Social Determinants of Health Discussion Paper 2 (Policy and Practice), Geneva: WHO.

Willet, W.C., Sacks, F., Trichopoulou, G., Drescher, G., Ferro-Luzzi, A. Helsing, E. and Trichopoulus, D. (1995). Mediterranean diet pyramid: a cultural model for healthy eating. Am J Clin Nutr, 61(6): 1402S-1406S.

Willson, A.E., Shuey, K.M., Elder, G.H. (2007). Cumulative advantage processes as mechanism of inequality in life course health. Am. J. Sociol. 112 (6), 1886e1924

$\mathrm{Xu}, \mathrm{X}$. eKaestner, R. (2010). The business cycle and health behaviors. National Bureau of Economic Research, Working Paper No. 15737. 